\title{
Reforma de la ley federal del trabajo en México : la consolidación de un modelo productivo financiarizado
}

Réforme de la loi fédérale du travail au Mexique: la consolidation d'un modèle productif financiarisé.

Reform of the Federal Labor Law in Mexico: The Consolidation of a Financialized Productive Model

\section{Élodie Ségal}

\section{OpenEdition}

\section{Journals}

Edición electrónica

URL: https://journals.openedition.org/ideas/852

DOI: $10.4000 /$ ideas.852

ISSN: 1950-5701

\section{Editor}

Institut des Amériques

\section{Referencia electrónica}

Élodie Ségal, «Reforma de la ley federal del trabajo en México : la consolidación de un modelo productivo financiarizado», IdeAs [En línea], 5 | 2015, Publicado el 18 junio 2015, consultado el 18 octubre 2022. URL: http://journals.openedition.org/ideas/852 ; DOI: https://doi.org/10.4000/ideas.852

Este documento fue generado automáticamente el 18 octubre 2022. 


\title{
Reforma de la ley federal del trabajo en México : la consolidación de un modelo productivo financiarizado
}

\author{
Réforme de la loi fédérale du travail au Mexique : la consolidation d'un modèle \\ productif financiarisé. \\ Reform of the Federal Labor Law in Mexico: The Consolidation of a Financialized \\ Productive Model
}

Élodie Ségal

\section{Introducción}

1 México vivió un momento crucial en torno a la votación de la reforma de la ley federal del trabajo. A finales de 2012, una preocupación fuerte, social y política, en torno a los cambios impulsados por la reforma movió el país. A través de numerosos debates y de movilizaciones colectivas podemos observar que tanto las posturas a favor como en contra de la propuesta emitida por el Partido Acción Nacional ${ }^{1}$ (PAN) en octubre de 2012 contemplan la noción de "trabajo digno " y, por ende, se preocupan por valores como el cuidado del otro en una sociedad desfigurada por las desigualdades .

El PAN en su propuesta define el « trabajo digno » 0 « decente » como aquél en el que se respeta plenamente la dignidad humana del trabajador; no existe discriminación por origen étnico o nacional, género, edad, discapacidad, condición social, condiciones de salud, religión, opiniones, preferencias sexuales o estado civil; se tiene acceso a la seguridad social y se percibe un salario remunerador; se recibe capacitación continua para el incremento de la productividad y del bienestar del trabajador, y se cuenta con condiciones óptimas de seguridad e higiene para prevenir riesgos de trabajo. ${ }^{2}$

3 Sin duda, la reforma propone un reto en la articulación de la democratización y de la competitividad. De hecho, presenta iniciativas destacables como la protección de los derechos de los trabajadores domésticos-en su mayoría trabajadoras-, el 
establecimiento de la capacitación laboral, el otorgamiento de licencias por paternidad, la reglamentación de los trabajadores atípicos - migrantes, trabajadores que ejercen desde el hogar, etc. -, la erradicación tanto de las represalias laborales a las mujeres embarazadas como del hostigamiento sexual en el ámbito laboral, entre otros temas. Estas modificaciones denotan las transformaciones sociales que se están viviendo en México.

4 Sin embargo, queremos cuestionar las vías posibles que nos propone esta reforma en cuanto al reconocimiento del trabajador en derecho. ¿Qué modelo de competitividad puede permitir un proyecto tan ambicioso? ¿Qué tipo de productividad institucionaliza la reforma de la ley federal del trabajo, individual o colectiva? ¿Qué visión del ser humano necesitamos para constituir una sociedad basada en los valores de dignidad, de solidaridad y del cuidado del otro?

La tesis que desarrollaremos se basa en una lectura de la reforma de la ley federal del trabajo en términos de individualización de la relación salarial y de la racionalización de las subjetividades (Ségal E., 2006: 21). De acuerdo con nuestra observación, el surgimiento de un nuevo modelo productivo orientado a la finanza ejerce un impacto directo sobre la desregulación de los derechos acordados para los trabajadores.

\section{Reforma laboral, modernización del país y competitividad}

\section{Política de "modernización" de México}

Un primer propósito alarmante de la reforma laboral observado, entre otros por Edgar Belmont, es la justificación de ciertos cambios desfavorables para los trabajadores tomando como argumento la modernización del país (Belmont E., 2012). Este tema aparece en México mucho antes de la reforma laboral, desde el 2 de septiembre de 2009, y está propuesto por el poder ejecutivo federal. La idea es modernizar el país y también modernizar los sectores estratégicos y atractivos en la denominada "nueva economía", que en México son los sectores energéticos y de telecomunicación. Pero detrás de la idea de modernización se esconde la determinación de encaminar el país hacia el libre mercado y la desregulación, promovida por intereses de empresarios y políticos, así como de algunos países europeos o de Estados Unidos. Las fuerzas que luchaban en contra de la lógica neoliberal, oponiéndose a la desregulación del país y a la privatización del sector público mexicano, se encuentran gravemente debilitadas, como nos muestra la disolución del sindicato de Luz y Fuerza del Centro por decreto el 11 de octubre del 2009. Así pues, el concepto de modernización no hace referencia a los valores de equidad y de dignidad, sino a la valoración de la lógica financiera que define los criterios de gestión de los trabajadores.

De manera general, la mayoría de los países industrializados han impulsado esta dinámica. En América Latina, a partir de los años 1980, países como Brasil y Argentina introdujeron cambios más drásticos que los que actualmente se debaten en la reforma laboral de México, e hicieron así patente una política de precarización laboral. El diagnóstico posterior ha mostrado que no se creó más empleo como tal, sino más empleo precario. En el caso argentino, después de diez años de flexibilización y de precarización laboral podemos observar un aumento del empleo desprovisto de seguro social. Un bloque importante de los países latinoamericanos decidieron dar un giro 
drástico y rechazar una visión de la competitividad fundada en la individualización de la relación salarial. La competitividad puede ser articulada a partir de valores diferentes a la rentabilidad financiera a corto plazo, como la protección social y la compartición del trabajo y de sus beneficios.

\section{La competitividad mexicana}

8 El segundo propósito que nos preocupa reside en la manera cómo la reforma laboral aborda la noción de competitividad, articulándose en torno a los valores de la denominada "nueva economía". Así pues, los trabajadores deben desarrollar nuevas competencias laborales que les permitan ser adaptables e innovadores en un mercado flexible y móvil. En este sentido el outsourcing o la subcontratación - uno de los puntos más polémicos de la reforma laboral, cuya reglamentación es sin duda necesaria apoya un modelo productivo que considera la mano de obra como un costo y no como un valor para la economía. La financiación de la economía ha destruido un valor del trabajo que debería ser pensado de manera solidaria y compartida. Compartir el trabajo significa pensar en una sociedad en la cual cada individuo puede acceder a una actividad remunerada con garantía de protección social, pero a su vez quiere decir también compartir los beneficios del trabajo.

9 Enrique de la Garza Toledo ha denunciado una visión de la competitividad basada en una mano de obra a bajo costo (De la Garza E., 1999). Datos de 2006 y 2007 de la OIT (OIT, 2008a ; 2008b) muestran que México tiene el sueldo mínimo más bajo de América Latina. Según Rhenals M. Remberto, en América Latina

Un examen de las tendencias recientes muestra que el salario mínimo, como porcentaje del salario medio, ha venido aumentando en la última década en los países de la región, con excepción de México. En 1995-1997 el salario mínimo relativo promedio de América Latina oscilaba alrededor del $40 \%$, mientras que en 2006-2007 se situó en un 50,7\%. En Argentina se observa el mayor aumento (45 puntos porcentuales); en Panamá, Chile, Perú, Uruguay, Brasil y Paraguay oscila entre 8 y 24 puntos porcentuales; permanece relativamente estable en Venezuela, Colombia, Costa Rica y Honduras, aunque en los tres primeros está en niveles cercanos o superiores al $50 \%$, y se reduce en México y El Salvador.$^{3}$ México sigue teniendo el sueldo mínimo más bajo de todos ellos.

\section{Individualización de la relación salarial}

11 Nosotros hablaremos además de la individualización de la relación salarial presente de manera transversal en la reforma laboral, o para ser más precisos, denunciaremos la articulación entre remuneración y productividad individual. La individualización de la relación salarial va en contra de las garantías colectivas de los trabajadores y se genera en la remuneración y en la evaluación del trabajo, que en la reforma del trabajo están sujetas a criterios subjetivos determinados por los propios directivos. Ellos acaban definiendo, según un juicio personal, la productividad de cada trabajador $\mathrm{y}$, en consecuencia, deciden cuál debe ser su remuneración. En este vínculo creado, los trabajadores son considerados como objetos en la relación salarial. Un sistema más equilibrado concibe la competitividad de manera colectiva y establece el sueldo de los trabajadores basándose en indicadores estables y colectivos garantizados por el Estado, como son el título profesional o la experiencia y el puesto laboral. Por este motivo 
queremos llamar la atención sobre algunos puntos olvidados o no resueltos satisfactoriamente en la reforma laboral, como por ejemplo la falta de seguro de desempleo, el retraso de la jubilación, el paso de un contrato colectivo a otro individual y temporal, la cosificación del trabajador como mercancía, la facilitación del despido al no contemplar liquidación o finiquito alguno, etc.

\section{Regulación neofordiana de acumulación del capital, la flexibilidad externa y la movilización por los mercados financieros}

\section{Un nuevo modelo productivo}

12 Es posible identificar tres posturas académicas que analizan las formas que adoptan las regulaciones productivas dentro del capitalismo contemporáneo. La primera, hoy ampliamente compartida, sostiene que existiría una pluralidad de modelos dominantes (Zarifian P., 2001) o, todavía más, una mezcla de modelos productivos (De la Garza, 1999). La segunda considera que el taylorismo, lejos de estar muerto, todavía dominaría la empresa. Los autores que sostienen esta posición resaltan las adecuaciones del taylorismo con, por ejemplo, el establecimiento de una "gestión por competencias » (Veltz P., 2000) o más generalmente la irrupción de nuevos modos de administración (Sennett R., 1998). La última postura subraya la estabilización de un nuevo modelo y de una forma renovada de regulación productiva. Dentro de esta posición, se insiste sobre la difusión del toyotismo (Boyer R., Durand J.-P., 1998) o sobre el desarrollo de variantes, como el modelo importado de las finanzas, calificado como «neoliberal » (Coutrot T., 1999), « flexible » (Aglietta M., 2005) o « financiarizado » (Boyer R., 1999).

Robert Boyer subraya que el nuevo modelo productivo posee cuatro características: "la descentralización de la producción", «la colectivización de la pericia », «asalariados calificados y adaptables» $\mathrm{y}$ "relaciones de trabajo cooperativas y favorables a la innovación ». La relación salarial que trae aparejada esta nueva organización productiva se funda sobre un compromiso de largo plazo entre los directivos y los asalariados en la cual « competencia laboral y lealtad se intercambian por una estabilidad en el empleo y/o un reparto de los resultados financieros de la empresa » (Boyer Robert, 1993 : 45). Mientras que frente a la ley del mercado todos los individuos dentro de la empresa deben cooperar con el buen ambiente y la armonía, la realidad experimentada en la empresa es diferente debido a que esta cooperación es impuesta por los mercados. Así pues, se pide a los individuos ser sujetos dentro de su trabajo, aún cuando ellos permanecen como objetos dentro de la relación salarial. objetos dentro de la relación salarial y a través de la remuneración como lo hemos visto en el caso de la reforma laboral en México.

El concepto de "cooperación forzada " propuesta por otro regulacionista, Thomas Coutrot, amplía esta idea al dar cuenta del régimen de movilización neoliberal. Este último se define como "un elemento central de la relación salarial. Consiste en un conjunto coherente de reglas que permiten tratar las incertidumbres que afectan la reproducción del capital individual y transformar la fuerza de trabajo en trabajo eficaz » (Coutrot T., 1998 : 12). Así, el régimen de movilización permite dar cuenta de las disposiciones establecidas por la empresa con el fin de interesar y comprometer su personal. Desde 1990, estas disposiciones han adquirido un gran poder. Según la Escuela 
de la Regulación, la precariedad laboral, pero también el aumento del desempleo y los despidos han estado acompañados del surgimiento de un nuevo modelo productivo. Este último ha privilegiado la comunicación, la confianza, la cultura de empresa, pero no ha abolido, sin embargo, los métodos coercitivos. Thomas Coutrot sostiene hoy en día que el concepto de « cooperación forzada " permite descubrir estas nuevas formas de coerción. Es a través de la cooperación, la cual parece natural e ineluctable dentro de la empresa neoliberal, que las obligaciones más macrosociales penetran los lugares de trabajo. Thomas Coutrot identifica esencialmente en los accionistas mayoritarios una presión que viene a transformar los colectivos productivos de la empresa.

Los mercados financieros representan una amenaza de sanción para los colectivos de trabajo que no alcancen las normas económicas que aquellos han fijado. Los mercados financieros imponen nuevas reglas organizacionales (particularmente en materia de la administración del personal) que afectan de cerca a los asalariados y su actividad de trabajo. Dominique Plihon (Plihon D., 2000:1) subraya también estas mutaciones: formula la hipótesis según la cual « se pueden explicar ampliamente ciertas mutaciones económicas y financieras observadas en Europa a finales del siglo XX a partir de este cambio de régimen macro financiero ». Desde los años 1980 se ha pasado de un régimen de endeudamiento (donde las empresas se financiaban con endeudamiento bancario) a uno de fondos propios. Esta nueva forma de financiamiento tiene dos consecuencias: por un lado, el ascenso de la inestabilidad financiera internacional y, por el otro, una relación cada vez más desigual entre el trabajo y el capital.

La financiarización de la economía puede manifestarse dentro de la empresa a través de políticas de accionariado. Pero queda difícil encontrar un vínculo directo entre el compromiso del trabajador y el accionariado asalariado. Al contrario, en general los asalariados tienen conciencia de no poseer un nuevo poder a través del accionariado asalariado. Dominique Plihon destaca que, de hecho, tres tipos de inversionistas poseen una parte considerable del capital, lo cual les permite remitir a un segundo plano el desarrollo de la actividad y del empleo con el fin de privilegiar la creación de valor accionario : los fondos de pensión anglosajones, los fondos mutuales y las compañías de seguros. Es a través de las reglas de administración que estos accionistas principales intentan imponer a los administradores y directivos de recursos humanos el valor de las acciones. Así, la rentabilidad financiera exigida por los accionistas se convierte en una prioridad central dentro de la empresa.

\section{Flexibilidad externa e interna}

17 El vínculo establecido por la escuela de la regulación entre, por un lado, la movilización de los trabajadores y, por el otro, la flexibilidad externa, en general, y aquella de la financiarización, en particular, nos parece estar atravesado por un momento de estancamiento. El vínculo entre los esquemas macroeconómicos y lo vivido por los asalariados debe pasar por el análisis de la dimensión organizacional y humana del trabajo.

18 Al formalizar una organización estandarizada que facilita a la vez la actividad asalariada para las poblaciones insuficientemente calificadas y permite un incremento de la productividad individual, el sistema de organización tayloriano marca una ruptura con el modelo del oficio 4 . Sin embargo, esta organización del trabajo ha suscitado graves conflictos sociales precisamente debido a que no toma en cuenta la 
realidad de la actividad productiva, la cooperación efectiva y el compromiso personal del trabajador. Las numerosas incertidumbres y las fallas demandaban una gran destreza por parte de los obreros, los cuales no estaban reconocidos dentro de las organizaciones partiendo del principio de la separación entre las tareas de concepción y la ejecución. El reconocimiento incompleto de la actividad de los obreros condujo a actitudes tales como la resistencia obrera, el turnover o aún el ausentismo. Más allá de estas formas latentes de resistencia, se atestiguan también formas de resistencia más abiertas como huelgas, movilizaciones, conflictos sociales.

Es en respuesta a estos conflictos que, desde los años 1970, aparecen numerosas tentativas por enriquecer las tareas. El objetivo, inspirado en los experimentos del Tavistock Institut (Trist E., Higgin G., Murray H., et. al., 1963 ; Thorsrud, 1975), consiste en movilizar más la inteligencia en el trabajo para aumentar la productividad. Recurrir a grupos de expresión y a «talleres de calidad » ha acompañado el surgimiento de un nuevo modelo productivo : el toyotismo. Así, en Europa y los Estados Unidos, a mediados de los años 1980, los "talleres de calidad", los equipos autónomos, los "grupos proyecto" son formas de administración que vienen a sostener una organización copiada del modelo japonés. Esta administración se sostiene menos sobre la autoridad que sobre la autoactivación de las personas a través de la activación de flujos de producción y de la flexibilidad interna. Sin embargo, estas iniciativas internacionales se desmarcan del modelo japonés particularmente por la flexibilidad externa, aquella ligada al empleo descrito principalmente por la escuela de la regulación. La flexibilidad externa es coherente con la flexibilidad interna y la intensifica, característica del flujo tenso. De esta manera, se puede exponer la tesis de la movilización de la mano de obra a través de la racionalización de la subjetividad, la cual reintegra y supera al toyotismo.

Los efectos del flujo tenso sobre la actividad del trabajo son analizados por Jean-Pierre Durand. Destaca el principio según el cual las obligaciones impuestas por el mantenimiento del flujo de producción participan en el compromiso de los asalariados : Si nos dedicamos [...] a analizar los efectos del flujo tenso sobre el trabajo, su primera consecuencia es la de movilizar a todos los asalariados; se puede hablar de la «naturalización de las obligaciones » en el sentido que las obligaciones (prescripciones, procedimientos, presiones de tiempo, emergencias, todo con una mano de obra reducida al mínimo) del sistema productivo no son controlados más por una sola persona - el jefe -, sino que están inscritas dentro de la necesidad de mantener la tensión del flujo productivo ${ }^{5}$. Este método, importado de Japón, se desmarca del fordismo en el sentido que permite repensar la productividad de una empresa fuera de las fronteras del consumo masivo.

Existen diferentes definiciones y diferentes aplicaciones del fordismo (Wood S., 1993), sin embargo, de manera general es posible decir que este sistema de producción responde a los siguientes criterios : estandarización de los productos, de las máquinas y de las tareas ; mano de obra especializada y no calificada ; separación entre las tareas de concepción y las de ejecución ; remuneración de acuerdo con la tarea y compensación económica de la monotonía.

23 La Escuela de la Regulación específica también algunos determinantes mayores de naturaleza macroeconómica, como el papel del Estado dentro de la administración de la demanda económica, de sindicatos fuertes y una integración vertical de la organización industrial. 
En oposición al modelo americano tradicional, el modelo postfordista o neofordista sostiene un principio de autonomía particular : la no restricción de puestos y de tareas, la producción organizada en grupos de trabajo responsables de múltiples tareas. El principio de flujo tenso prolonga e intensifica el flujo fordiano. En efecto, dentro de este último, los stocks anteriores y posteriores a un puesto de trabajo a lo largo del flujo de producción han conducido a caracterizarlo como un flujo presionado. Dentro de la organización fordista, el consumo de masas inevitablemente venía a sostener la producción en masa y las existencias, aun cuando eran importantes en cantidad, se vendían rápidamente. Dentro del flujo tenso, una obligación vino a desajustar este sistema : la exigencia de calidad y de producción justo a tiempo (ver la siguiente ilustración).

Flujo presionado y flujo tenso

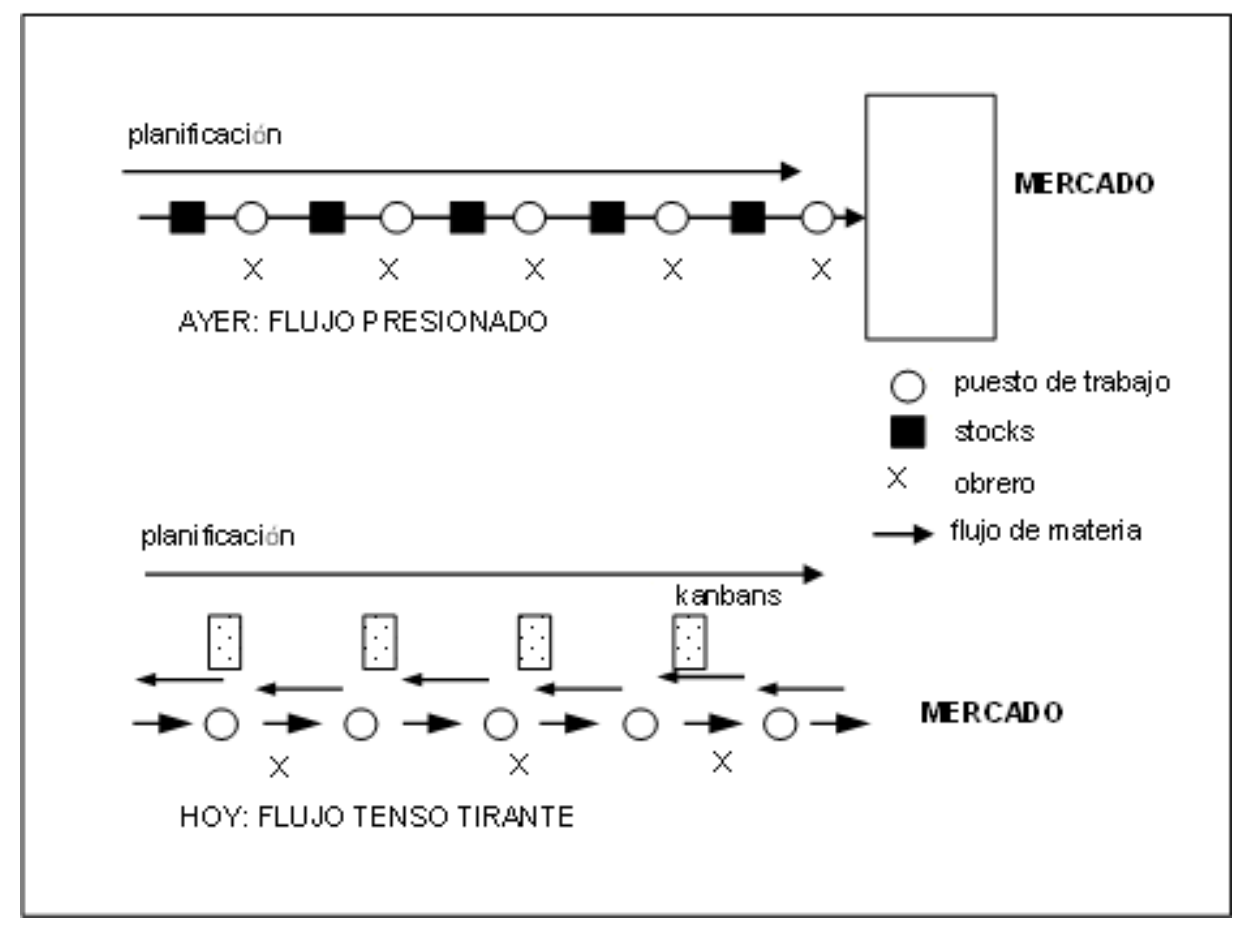

EL MODELO POSTFORDIANO ESTÁ CARACTERIZADO ASÍ POR UN PRINCIPIO DE TENSIÓN DEL FLUJO QUE SE HA CONVERTIDO EN UN FLUJO QUE ES TIRADO POR LA DEMANDA. ES GUIADO POR LOS PEDIDOS DEL CONSUMIDOR Y NO POR UN PRINCIPIO DE PRODUCCIÓN EN MASA.

FUENTE : JEAN-PIERRE DURAND (2000 : 55)

Es así como en la superación de la producción en masa, encontramos la especificidad del modelo japonés. La flexibilidad interna, la cual tiene como motor la investigación y la innovación, la renovación de productos, de procedimientos, de máquinas, etc., tiene como consecuencia hacer que las exigencias en términos de polivalencia y de policompetencia se apoyen sobre los asalariados. Jean-Pierre Durand (Durand J.-P., 2004) ha sintetizado los grandes componentes del modelo productivo postfordiano de acumulación del capital bajo la denominación de «nueva combinatoria productiva ». Esta expresión muestra cómo tres polos, la integración reticular, la generalización del flujo tenso y el modelo de la competencia laboral, interactúan para dar coherencia a un nuevo régimen de movilización de la mano de obra. El componente del flujo tenso permanece, según nosotros, como aquel a través del cual los otros dos, la integración reticular (integración entre investigación e industrialización, integración funcional) y 
el modelo de la competencia laboral, pueden articularse. Debido a que el flujo tenso permanece como el componente más materializable, actúa por lo tanto como una estructura sobre los otros dos elementos de la nueva combinatoria productiva.

\section{Modelo de la competencia laboral : la mundialización como motor de la individualización de la relación salarial}

\section{Economía del conocimiento y competencia laboral}

Este regreso al tema de la organización material de la producción nos permite ver cómo el modelo de la competencia laboral es un debate central en el sistema de producción emergente. En éste, la individualización de la relación salarial, como hemos visto en la primera parte sobre la reforma laboral, viene a destruir los colectivos de trabajo y de trabajadores.

De todas las perspectivas, las competencias laborales son consideradas como un imperativo frente al desarrollo de la economía del conocimiento y se han convertido en un punto de referencia central dentro de los distintos ámbitos del trabajo (Barnett R., 2001) como en la reforma de la ley federal del trabajo que ha votado México. En efecto, ellas son utilizadas para definir las normas de calificación de los trabajadores en términos de niveles y de contenidos que puedan reflejar las necesidades de las empresas : asimismo, ellas se traducen en el modo de administración de los recursos humanos. Las competencias corresponden a los "saberes ", los « saber hacer " y sobre todo a los "saber ser " de los trabajadores. Los «saber ser " concentran las nuevas exigencias de nuestra economía. Las organizaciones internacionales, como la Orgnización de Cooperación y Desarrollo Económico (OCDE), ejercen una gran influencia a este respecto (OCDE, 2003). En ambos lados del Atlántico, las reformas en marcha dentro del ámbito laboral presentan similitudes. En Europa, Estados Unidos, Canadá o en América Latina, los dispositivos de concertación impulsan el desarrollo normalizado de las competencias de la mano de obra más allá del empleo ocupado. Al mismo tiempo, en nombre del empleo, los modelos de administración de los recursos humanos proponen sistemas de remuneración y de motivación alrededor de las competencias individuales (Leonor C., Quiroz A.M., 1997).

La comparación a nivel internacional permite discernir las diferencias dentro de los modos de organización del trabajo, de los sistemas educativos, de las formas de clasificación del empleo y de las diversas apreciaciones que se forman respecto a la calificación. Así, Marc Maurice, François Sellier y Jean-Jacques Silvestre (Maurice M., Sellier F., Silvestre J.-J., 1982 : 355) analizaron el relativismo de la tecnología sobre las relaciones de trabajo. Los sistemas educativos, los sistemas de capacitación y las formas de organización juegan un papel de mediación entre las tecnologías y las relaciones de trabajo. Dentro de esta perspectiva, es común distinguir diferentes enfoques de la competencia. Sin oponernos a estas perspectivas, desarrollaremos la hipótesis según la cual la « lógica competencia » y, sobre todo, la individualización de la relación salarial que ella induce, rebasan las fronteras nacionales (Ségal E., 2005). En efecto, la internacionalización de los intercambios permite dificilmente circunscribir las preocupaciones propias a un sólo país. Más precisamente, las concepciones americanas de la competencia (búsqueda de una adecuación entre las conductas de los asalariados y 
las políticas de la empresa) se encuentran más allá de la diversidad de los modelos presentes en el ámbito internacional. La mundialización, la tendencia creciente a recurrir a la "externacionalización » de los sitios de producción y los cambios del cuadro legal de numerosos países contribuyen a difundir las estrategias elaboradas por los diferentes grupos empresariales. La "gestión por competencias " ha sido trabajada en redes de investigación, en organizaciones sindicales y en redes de empresas multinacionales ${ }^{6}$. En este sentido, dos tradiciones se oponen : por un lado, Francia que promueve un reconocimiento del título profesional y, por el otro, los países anglosajones que proponen acercarse a criterios relativos a los individuos y a sus motivaciones (Stein R., 2003). En América Latina, los distintos países implementan los dos modelos. Por ejemplo, México posee instituciones centrales para la aplicación de la lógica de las competencias, como es el Consejo de Normalización y Certificación de Competencia Laboral ${ }^{7}$ (CONOCER). Sectorizado en la Secretaría de Educación, este Consejo había seguido hasta los años 2006 las políticas de certificación francesas alrededor de las calificaciones. Con esta concepción, Francia se encuentra en un compromiso y un proceso de regulación más colectivo; después de la Segunda Guerra Mundial, el Estado, los sindicatos y las organizaciones patronales negociaron alrededor de la calificación. Ella garantiza un compromiso alrededor del puesto ocupado, del título y de la remuneración. La regulación de estos tres actores fuertes permitieron certificar algunos intereses:el Estado atestiguó el título profesional; los sindicatos, las condiciones de trabajo y el sueldo mínimo; y las organizaciones patronales, la estandarización de los puestos y la racionalización de la organización del trabajo. Hoy el CONOCER trabaja con una lógica anglosajona más conductista, lo cual es una tendencia internacional que se ha generalizado, como lo analizaremos en el apartado siguiente.

La investigación europea de François Geffroy y René Tijou (Geffroy F., Tijou R., 2002) sugiere el predominio del modelo conductista en los países europeos. Así, entre las treinta empresas estudiadas en esta investigación, dos de cada tres partes afirman llevar a cabo una "gestión por competencias» fundada en las competencias individuales. Solamente una de cada tres empresas estudiadas centra su funcionamiento en la gestión por el oficio. Además, en la mayoría de las empresas estudiadas se hace énfasis en las competencias conductistas. Para complementar las observaciones de esos dos autores y analizar lo que puede pasar en un contexto legal que desestabiliza la definición colectiva del trabajo y de su cuadro legal hemos estudiado la política de gestión por competencias laborales de veinte transnacionales (Xerox, Renault, PSA, Microelectronics, Coca Cola, Thomson Télévision, Ajinomoto Euro Aspartame, Entreprise Sollac, Pechiney, Total Fina Elf, Thales, Blédina, LU France, Danone Belgique, Danone México, Opavia, Danone Italia, Linneo, Usine de Sant Hilari, Rhodia, Ervaf); varias de ellas son muy activas en América Latina y en México. El resultado de nuestra investigación describe la misma constante que F. Geffroy y R. Tijou : las veinte empresas investigadas desarrollan competencias conductistas dentro de sus referenciales, pero también dentro de sus herramientas de evaluación.

\section{« Gestión por competencias » y mundialización}

31 Las empresas analizadas han decidido instalar una "gestión por competencias » para cumplir con los imperativos de desempeño. Estos últimos están directamente ligados a la generalización de un contexto económico globalizado. Según los directivos de las 
empresas analizadas, la mundialización de los intercambios se apoya sobre tres grandes imperativos : la competitividad en el mercado, el crecimiento y la creación de valor, así como las exigencias de los clientes. Estos tres imperativos dentro de la administración de la empresa son movilizados ampliamente por los grupos empresariales objeto de esta investigación. Dentro de estas prácticas, la « lógica competencia » debería permitir mantener, si no mejorar, su lugar en el mercado.

En primer lugar, el argumento de la competencia en el mercado frecuentemente ha sido abordado dentro de las entrevistas. El personal de Recursos Humanos (RH) entrevistado evoca, por un lado, la saturación de los mercados occidentales - Renault, L'Oréal, Usinor - y, por otro lado, la disminución de los precios de venta - Arcelor. La solución que se vislumbra es la creación de alianzas y de asociaciones. En efecto, las diferentes fusiones han fortalecido a las multinacionales cada vez más dentro de los mercados, pero al mismo tiempo han debilitado a las empresas. Esta nueva regla del mercado se impone de diversas formas. Arcelor, por ejemplo, desarrolla dos tipos de estrategia : la búsqueda de asociaciones complementarias y el acompañar a grandes clientes en el plano internacional. También atestiguamos la alianza a través del control - Renault - y sobre todo la adquisición (por las grandes empresas como Danone), es decir, la fusión. En este caso, la competitividad del mercado es percibida por los practicantes con los que nos reunimos, como la justificación de un cambio ineluctable. En este caso, la Dirección de Recursos Humanos (DRH) de Arcelor recuerda la crisis petrolera de finales de los años $1970 \mathrm{y}$ el fin del control de precios por parte del Estado. Estos factores son vividos como el motor de cambio que hoy debe apoyarse en el factor humano.

En segundo lugar, el crecimiento y la creación de valor son dos argumentos utilizados por numerosas empresas preocupadas por su expansión internacional. Ellas desean convertirse en líderes en su mercado y, para ello, frecuentemente recurren a proyectos de fusión-adquisición. Esta voluntad de crecimiento es reivindicada por empresas tales como L'Oréal o ST Electronics. En la búsqueda del crecimiento se agregan preocupaciones por la creación de valor. Estas preocupaciones se presentan en empresas esencialmente enfrentadas a la competencia del mercado internacional, tales como Danone y Arcelor. En estos dos casos, el peso de los accionistas es colocado en principio sobre las decisiones de los dirigentes. Aquí, el argumento clave para el establecimiento de una "gestión por competencias» es el avance tecnológico. En efecto, este último es presentado como una seria ventaja tanto para el crecimiento como para la creación de valor. La búsqueda de competencias clave es pues central debido a que ella permite desarrollar la innovación tecnológica deseada. Por ejemplo, Danone y Arcelor buscan la "policompetencia». Sin embargo, la innovación tecnológica pasa también por la búsqueda de innovaciones globales por parte de la empresa. En estos dos casos modelo, para buscar competencias clave y una tecnicidad global, las multinacionales implementan una doble estrategia : desarrollan actividades claves y subemplean otras. El caso de Renault es significativo. Si por largo tiempo ha funcionado en el aseguramiento de la totalidad de la producción de autos, hoy Renault ha recurrido a la compra de insumos y piezas a otras empresas. Renault funciona hoy más sobre una lógica de red y de proyecto que debe ser coordinado, que sobre la lógica manufacturera tradicional. Dentro de esta lógica, ciertos oficios son privilegiados. Por ejemplo, la dirección desarrolla en particular los oficios relacionados con la ingeniería y con lo comercial. 

competencias». Esto puede pasar por el propósito de personalizar los productos Renault -, la adaptación al contexto sociodemográfico de una región - L'Oréal - o aún acompañar a grandes clientes al extranjero - Arcelor, ST Electronics. Dentro del sector de los servicios, es ampliamente utilizado el argumento de la satisfacción « de las exigencias de los clientes». Sin embargo, esas exigencias también se presentan en el sector industrial, a través de un doble sesgo : por un lado, la invención de una nueva cultura de la producción que incrementaría su productividad a favor del cliente y, por otro lado, por las grandes empresas multinacionales, el desarrollo de «proyectos competencias» orientados hacia la búsqueda de los trabajadores con el mejor desempeño. mostrarse flexibles a las diferentes expectativas, producir con calidad, someterse a diferentes normas de calidad»-DRH, Blédina. En el segundo caso, los asalariados deben apoyar las orientaciones estratégicas de su grupo. Este apoyo pasa por el cambio de las conductas. Arcelor privilegia, por ejemplo, la adaptación a nuevas lógicas de los oficios, una mayor adaptabilidad y capacidad de reacción-polivalencia, policompetencia. Thales desea orientar a sus trabajadores hacia una «cultura del cambio ", mientras que Danone desarrolla una « cultura de grupo » - corporate. Para las empresas analizadas, los clientes no dudan en hacer funcionar la competencia internacional sobre los precios, la calidad o aún sobre los plazos. La figura del cliente puede diferir poco en el caso de la subcontratación. Los subcontratistas, en efecto, son fuertemente tributarios de las empresas que les envían pedidos, de grandes multinacionales como Renault o Thales, debido a que aseguran la actividad principal de la fábrica. La pérdida de un gran cliente es frecuentemente asociada a la pérdida de la actividad global del sitio de producción.

La «gestión por competencias » privilegiadas en cada caso (búsqueda de crecimiento y creación de valor, competencia del mercado y de la satisfacción del cliente) favorece una estrategia de cambio apoyada en los hombres. Esta lógica es internacional y se inscribe en un contexto económico mundializado que unifica las estrategias de DRH presentes en el mercado y el cual también es facilitado por la mundialización de los sitios de producción.

\section{Conclusión : Reconocer el trabajador en derecho}

37 Una propuesta desarrollada por laboralistas franceses consiste en reconocer al trabajador en derecho. Esta conceptualización no se aborda desde una reforma laboral, sino desde un cambio en la constitución misma. La constitución actual mexicana fue escrita, en parte, tomando como ejemplo la francesa, la cual después de la revolución se había basado en el poder del dinero - en el valor unilateral de la moneda. En esta lógica, los trabajadores son todavía hoy en día considerados como un costo, al igual que las máquinas, y no como un valor en la contabilidad de las empresas. En el caso mexicano, el pago por hora - unidad de tiempo -, la falta de seguro de desempleo, el retraso de la jubilación, la facilitación del despido al no contemplar liquidación muestran la consideración del trabajador como un costo. La sociedad mexicana pierde el contrato colectivo cuando se contrata y sobre todo cuando se remunera el trabajo individualmente y temporalmente. Hay un riesgo de cosificar al trabajador como 
mercancía. Además, el despido no contempla « salarios caídos » lo que facilita cualquier despido. caso de sus sectores energéticos y de telecomunicación, hay un sentido universal y más noble en la actividad laboral. El trabajo va desde la realización personal hasta la realización colectiva. Esta unidad política tiene un sentido más complejo y profundo que los intereses económicos de sus miembros. En una sociedad como la mexicana llamamos la atención sobre la definición de «trabajo digno » visto como el cuidado del otro. Los debates sobre el voto de la reforma federal del trabajo permitieron pensar como preámbulo - en la protección de los derechos de los trabajadores domésticos, el establecimiento de la capacitación laboral, el otorgamiento de licencias por paternidad, la reglamentación de los trabajadores atípicos - migrantes, trabajadores que ejercen desde el hogar, etc. -, la erradicación tanto de las represalias laborales a las mujeres embarazadas como del hostigamiento sexual en el ámbito laboral, entre otros temas. Varios intelectuales trabajaron en la conceptualización de la noción de «trabajo digno ", llamada también el care. Es una preocupación ética para la prevención, la sanación, el cuidado del otro y la solidaridad, y a la vez representa un reconocimiento social y financiero de todas las categorías socio-profesional que dedican su vida laboral a este sector de actividad.

Además, numerosos intelectuales retomaron el proyecto de obtener un sueldo universal para todos con el fin de dar una propuesta más concreta a las tensiones provocadas por la crisis, y para conseguir mayor modernización, competitividad y solidaridad. En particular, Bernard Friot (Friot B., 2012) critica la articulación entre la remuneración y el empleo. En situación de desempleo esta concepción de la actividad laboral deja de lado a una parte muy importante de la población. Bernard Friot propone el sueldo de por vida articulando la remuneración a un estatus y a la persona, como es el caso para los jubilados o los funcionarios en Europa. Aun si esta concepción trae consigo numerosos límites según el país - por ejemplo en México el título y el estatus son una herramienta más de discriminación social-, nos permite pensar en la importancia de la negociación de derechos y de garantías individuales y colectivas alrededor de la remuneración. No permitir la individualización de la relación salarial que trae consigo el modelo productivo característico de la financiarización de la economía, es un verdadero reto para América Latina. 


\section{BIBLIOGRAFÍA}

Aglietta, Michel, Macroéconomie financière. 2, Crises financières et régulation monétaire, $4^{\mathrm{a}}$ ed., Paris, La Découverte, 2005.

Barnett, Ronald, Los límites de la competencia, Barcelona, GEDISA, 2001.

Belmont, Edgar, « Luz y fuerza del centro : ejes del conflicto entre el sindicato mexicano de electricistas y el gobierno federal », Estudios sociológicos : México, D.F. : El Colegio de México, Centro de Estudios Sociológicos, vol. 30, n 89, 2012, p. 331-365.

Boyer, Robert, « Comment émerge un nouveau système productif ? ", in Jean-Pierre Durand (dir.), Vers un nouveau modèle productif ?, Paris, Syros, 1993, p. 31-92.

Boyer, Robert, « Le politique à l'heure de la mondialisation et de la finance. Le point sur quelques recherches régulationnistes », L'Année de la régulation, vol. 3, Paris, La Découverte, 1999, p. 13-77.

Boyer, Robert y Durand, Jean-Pierre, L'Après-fordisme, 2ª ed., Paris, Syros, 1998.

Coutrot, Thomas, L'entreprise néo-libérale, nouvelle utopie capitaliste?, Paris, Éd. la Découverte, 1998.

Coutrot, Thomas, Critique de l'organisation du travail, Paris, La Découverte, 1999.

De la Garza, Enrique, « Epistemología de los Modelos de Producción » in Enrique De la Garza (dir.), Los Retos Teóricos de los Estudios de Trabajo Hacia el Siglo XXI, Buenos Aires, CLACSO, 1999.

Durand, Jean-Pierre, « Flux tendu et modèle de la compétence : une révolution tranquille », Issues, $\mathrm{n}^{\circ}$ 55-56, 2000, p. 47-70.

Durand, Jean-Pierre, La chaîne invisible : travailler aujourd'hui : flux tendu et servitude volontaire, Paris, Le Seuil, 2004.

Friot, Bernard, L'enjeu du salaire, Paris, La Dispute, 2012.

Geffroy, François et René Tijou, Le management des compétences dans les entreprises européennes, les différentes approches, Paris, INSEP Consulting Éditions, 2002.

Leonor, Cariola H. y Quiroz, Ana María, « Competencias generales, competencias laborales y currículum » en Marta Novick, Mara Bartolomé et Mariana Buceta et. al. (dir.), Nuevos puestos de trabajo y competencias laborales. Un análisis cualitativo en el sector metalmecánica argentino, Montevideo, CINTERFOR, 1997, p. 51-77.

« Ley Federal del trabajo », Editorial SISTA, Mexico, 2013.

Maurice, Marc, Sellier, François y Silvestre, Jean-Jacques, Politique d'éducation et organisation industrielle en France et en Allemagne : essai d'analyse sociétale, Paris, PUF, 1982.

OCDE, « Primeros resultados de PISA 2003, Resumen ejecutivo », http://www.oei.es/quipu/ mexico/informe_pisa2003.pdf. Página consultada el 12 de agosto de 2007.

Organización Internacional de Trabajo - OIT, Evolución de los salarios en América Latina 1995-2006, 2008, Evolución de los salarios en América Latina 1995-2006 [OIT]. Página consultada el 12 de marzo de 2015.

Organización Internacional de Trabajo - OIT, Global Wage Report 2008 / 09, Minimum wages and collective bargaining : towards policy coherence . International Labor Office, Genève, 2008. http:// www.ilo.org/wcmsp5/groups/public/@dgreports/@dcomm/documents/publication/ wcms_100786.pdf. Página consultada el 27 de mayo de 2015. 
Plihon, Dominique, «L'économie de fonds propres : un nouveau régime d'accumulation financière ", in François Chesnais, Dominique Plihon (dir.), Les pièges de la finance mondiale, Paris, Syros, 2000.

Remberto, Rhenals M., « ¿ Es alto el salario mínimo en Colombia ? : una comparación internacional », Perfil de Coyuntura Económica, vol. 13, 2009, p. 97-145.

Ségal, Élodie, Les compétences dites "relationnelles" : quel contenu, quel apprentissage, quelle place?, Ministère de l'Éducation Nationale, de l'Enseignement Supérieur et de la Recherche, Paris, Coll. CPC documents, 2005.

Ségal, Élodie, Le dévoiement des "savoir-être". Usages sociaux et managériaux des compétences en entreprise, Thèse de doctorat en sociologie, Université d'Evry Val d'Essonne, 2006.

Sennett, Richard, The Corrosion of Character : The Personal Consequences of Work in the New Capitalism, New York, Norton, 1998.

Stein, Rokkan, « Working Paper 26-2003», Centre for Social Studies, Oslo, http:// www.rokkansenteret.uib.no/rPub/files/60_N26-03-Drexel.pdf. Página consultada el 27 de mayo 2015.

Thorsrud, Einar, « La démocratisation du travail et le processus de transformation de l'organisation », Sociologie du travail, vol. 27, n 3, 1975.

Trist, Eric, Higgin, Garth.W., Murray, Hugh, et.al., Organizational choice capabilities of groups at the coal face under changing technologies, London, Tavistock Publications, 1963.

Veltz, Pierre, Le nouveau mode industriel, Paris, Gallimard, 2000.

Wood, Stephen, « Le modèle japonais : postfordisme ou japonisation du fordisme ? ", in JeanPierre Durand (dir.), Vers un nouveau modèle productif?, Paris, Syros, 1993, p. 93-123.

Zarifian, Philippe, Le modèle de la compétence : trajectoire historique, enjeux actuels et propositions, Paris, Éditions Liaisons, 2001.

\section{NOTAS}

1. Los tres partidos políticos más importante de México, el Partido de la Revolución Democrática (PRD), el Partido Revolucionario Institucional (PRI) y el Partido Acción Nacional (PAN), trabajaron desde 2009 en sus propias reformas de la ley federal del trabajo. Desde julio hasta diciembre del 2012, el país estaba en una transición de poder político debido a las elecciones - de julio 2012 -, que otorgó la presidencia del país a Enrique Peña Nieto, representante del PRI. Desde entonces el PRI viene a sustituir al PAN. En esta transición será la propuesta del PAN la que será retomada y votada.

2. Ver Ley Federal del trabajo (2013: 15).

3. Ver Remberto (2009).

4. Entendemos por «modelo del oficio » o « lógica del oficio » a la forma de organización de la producción donde la actividad del trabajo es completa, es decir que la actividad laboral no ha pasado por la división y la separación de las tareas.

5. Ver Durand (2000:60).

6. Ejemplos de redes influyentes: en Francia, organizaciones patronales: objectif compétence MEDEF Démarche compétence - Une ressource web ; en América Latina, consultores : Portafolio Consultores E.A.T. Consultores, NTIC, Diplomados, Congresos, E- 
Learning, Blended Learning, Competencias, Investigacion; Gobierno británico : Department for Business, Innovation \& Skills - GOV.UK ; OCDE : OCDE - OCDE.

7. http://www.conocer.gob.mx/. El CONOCER es una organización integrada por trabajadores, empresarios, educadores, capacitadores y el gobierno federal que impulsa la competencia laboral certificada. El CONOCER impulsa el desarrollo continuo de los trabajadores mediante la evaluación y la certificación de sus conocimientos, habilidades y destrezas tomando como base los estándares de calidad que deben de cubrir en su desempeño y orientar la educación y la capacitación hacia las necesidades de los mercados productivo y laboral.

\section{RESÚMENES}

A finales de 2012, México vota una reforma de la ley federal del trabajo. Tanto las posturas a favor como en contra de la propuesta originalmente emitida por el Partido Acción Nacional (PAN) en octubre de 2012, contemplan la noción de "trabajo digno" y, por ende, se preocupan por valores como el cuidado del otro en una sociedad desfigurada por las desigualdades. Sin duda, la reforma propone un reto en la articulación de la democratización y de la competitividad. Sin embargo, cuestionamos las vías posibles que nos propone esta reforma en cuanto al reconocimiento del trabajador en derecho. ¿Qué modelo de competitividad puede permitir un proyecto tan ambicioso ? ¿Qué tipo de productividad institucionaliza la reforma de la ley federal del trabajo, individual o colectiva? ¿Qué visión del ser humano necesitamos para constituir una sociedad basada en los valores de dignidad, de solidaridad y del cuidado del otro ? La tesis que desarrollamos se basa en una lectura de la reforma de la ley federal del trabajo en términos de individualización de la relación salarial y de la racionalización de las subjetividades. De acuerdo con nuestra observación el surgimiento de un nuevo modelo productivo orientado hacia la finanza ejerce un impacto directo sobre la desregulación de los derechos acordados para los trabajadores.

Fin 2012, le Mexique vote une réforme de sa loi fédérale du travail. Les différents débats autour de la proposition initialement émise par le Parti d'Action Nationale (PAN) en octobre 2012, envisagent la notion de «travail décent » et, par là même, se centrent sur des valeurs telles que « la prise en charge » et le « soin » dans une société défigurée par les inégalités.

Sans aucun doute, la réforme propose un défi dans l'articulation de la démocratisation et de la compétitivité. Toutefois, nous interrogeons les moyens concrets proposés par cette réforme autour de la reconnaissance des droits des travailleurs. Quel modèle de compétitivité peut permettre un projet aussi ambitieux? Quel modèle productif institutionnalise-t-il cette reforme, individuelle ou collective? De quelles approches avons-nous besoin pour construire une société fondée sur des valeurs de dignité, de solidarité et d'altruisme ? La thèse que nous développons est basée sur une lecture de la réforme de la loi fédérale du travail en termes d'individualisation de la relation salariale et de rationalisation de la subjectivité des travailleurs. Selon notre observation, l'émergence d'un nouveau modèle productif financiarisé a un impact direct sur la déréglementation des droits acquis par les travailleurs.

End 2012, Mexico carried out a reform of the federal labor law. The notion of «decent work » adopted was able to obtain political conscensus, while taking into account such values such as «welfare» and «healthcare» in a society distorted by huge inequalities. This reform 
undoubtedly represents a challenge to the articulation between democratization and competitiveness. However, the concrete means provided by this reform concerning the recognition of workers' rights should be questioned: what kind of model of competitiveness may allow such an ambitious project? What kind of production model - individual or collective - is capable of institutionalizing this reform? What kind of approaches are needed to build a society based on dignity, solidarity and altruism? Our thesis is based on an understanding of the federal labour law reform in terms of individualization of wage labour and of rationalization of workers' subjectivity. According to our observation, the emergence of a new financiarized production model has a direct impact on the deregulation of workers' vested rights and benefits.

\section{ÍNDICE}

Mots-clés: droit du travail, modèle productif, travail décent, Mexique, modernisation, compétitivité, financiarisation.

Palabras claves: reforma laboral, modelos productivos, trabajo decente, México, modernización, financiarización, competitividad.

Keywords: labor reform, productive model, decent work, Mexico, modernization, financialized model, competitiveness.

\section{AUTOR}

\section{ÉLODIE SÉGAL}

Profesora Investigadora del Departamento de Estudios Institucionales de la UAM Cuajimalpa. Asociada al Centro Pierre Naville, Francia. segalelodie@yahoo.com. 